\title{
A COMPARISON OF COSMOLOGICAL HYDRODYNAMIC CODES
}

\author{
Hyesung Kang ${ }^{1,2}$, Jeremiah P. Ostriker ${ }^{1}$, Renyue Cen ${ }^{1}$, Dongsu Ryu ${ }^{1,3}$ \\ Lars Hernquist ${ }^{4}$, August E. Evrard ${ }^{5}$, Greg L. Bryan ${ }^{6}$ and Michael L. Norman ${ }^{6}$
}

Nov 5, 1993

\author{
Submitted to the Astrophysical Journal \\ ${ }^{1}$ Princeton University Observatory, Princeton, New Jersey 08544 \\ 2 mailing address: Department of Earth Sciences, \\ Pusan National University, Pusan, Korea \\ ${ }^{3}$ mailing address: Department of Astronomy and Space Science, \\ Chungnam National University, Daejun, Korea \\ ${ }^{4}$ Board of Studies in Astronomy and Astrophys, \\ University of California,Santa Cruz, California. \\ ${ }^{5}$ Department of Physics, University of Michigan, Ann Arbor, MI 18109 \\ ${ }^{6}$ National Center for Supercomputing Applications, Urbana, Illinois 61801
}




\section{ABSTRACT}

We present a detailed comparison of the simulation results of various cosmological hydrodynamic codes. Starting with identical initial conditions based on the Cold Dark Matter scenario for the growth of structure, with parameters $h=0.5, \Omega=\Omega_{b}=1$, and $\sigma_{8}=1$, we integrate from redshift $z=20$ to $z=0$ to determine the physical state within a representative volume of size $L^{3}$ where $L=64 h^{-1} \mathrm{Mpc}$. Five independent codes are compared: three of them Eulerian mesh based and two variants of the Smooth Particle Hydrodynamics "SPH" Lagrangian approach. The Eulerian codes were run at $N^{3}=\left(32^{3}, 64^{3}, 128^{3}\right.$, and, $\left.256^{3}\right)$ cells, the SPH codes at $N^{3}=32^{3}$ and $64^{3}$ particles. Results were then rebinned to a $16^{3}$ grid with the expectation that the rebinned data should converge, by all techniques, to a common and correct result as $N \rightarrow \infty$.

We find that global averages of various physical quantities do, as expected, tend to converge in the rebinned model, but that uncertainties in even primitive quantities such as $\langle T\rangle,\left\langle\rho^{2}\right\rangle^{1 / 2}$ persists at the $3 \%-17 \%$ level after completion of very large simulations. The two SPH codes and the two shock capturing Eulerian codes achieve comparable and satisfactory accuracy for comparable computer time in their treatment of the high density, high temperature regions as measured in the rebinned data; the variance among the five codes (at highest resolution) for the mean temperature (as weighted by $\rho^{2}$ ) is only $4.5 \%$. Examined at high resolution we suspect that the density resolution is better in the SPH codes and the thermal accuracy in low density regions better in the Eulerian codes. In the low density, low temperature regions the SPH codes have poor accuracy due to statistical effects, and the Jameson code gives temperatures which are too high, due to overuse of artificial viscosity in these high Mach number regions.

Overall the comparison allows us to better estimate errors, it points to ways of improving this current generation of hydrodynamic codes and of suiting their use to problems which exploit their individually best features.

subject headings: cosmology: theory - hydrodynamics - methods: numerical 


\section{INTRODUCTION}

Numerical simulations in cosmology have become powerful tools for the investigation of the quantitative consequences of the various (and proliferating) scenarios for the growth of structure and also for relating observational results concerning galaxy distributions back to more fundamental characterizations of the universe. Until fairly recently the method of choice was (after adopting some set of initial conditions) to integrate forwards in time the equations of motion for a set of collisionless particles in a fluctuating gravitational field, which itself is determined self consistently by the solution of Poisson's equation, given the contemporaneous density distribution determined by the particle positions. Examples of this method are the papers of Davis, Efstathiou, Frenk, and White (in various combinations, e.g. 1985a,b), Melott and colleagues (e.g. Centrella et al. 1980), Bertschinger and colleagues (e.g. Bertschinger \& Gelb 1991), Villumsen and colleagues (e.g. Brainerd \& Villumsen 1992) and others. The methods are highly developed and many important results have been obtained.

But, ultimately, when we observe the universe, we are typically observing the baryonic component, even if most of the matter resides in a collisionless "dark" component, which is treated appropriately by the techniques noted above. The X-ray emitting gas in clusters of galaxies, the Lyman alpha clouds, the hot or moving gas responsible for secondary fluctuations of the microwave background, and the gas from which galaxies were formed - this material - satisfies the classic Euler equations of hydrodynamics. If we wish to model these phenomena, we must solve those equations as well as the collisionless equation for the dark matter component (should such exist).

Several techniques have evolved for treating the gaseous component. One family, which traces itself back to the N-body methods familiar to astronomers, is called "Smooth Particle Hydrodynamics". Here one follows representative mass elements by methods analogous to classical dynamics but attaches thermodynamic variables (such as pressure) to the particles, so their motion can be affected by positions of other 
nearby particles (pressure gradients), and one has the possibility of treating, in some approximation, shocks where fluid elements moving in a given direction overtake and collide with other fluid elements, the collision transforming relative fluid velocity into thermal energy.

This is not the place for a technical exposition concerning methods. For a detailed presentation one should refer to Hernquist \& Katz (1989) for the SPH method utilized in this paper and designated TSPH and refer to Evrard (1988) for the Evrard version which is designated PSPH hereafter. The TSPH code uses the Tree method (Barnes \& Hut 1986) and PSPH uses the $\mathrm{P}^{3} \mathrm{M}$ (Efstathiou \& Eastwood 1981) method to calculate the gravitational field. The SPH methods are intrinsically Lagrangian in nature in that they follow individual fluid elements. This has the advantage that it puts the computational resources where they are needed most. Needless to say there are also attendant disadvantages to this method, one of which is that the thickness of a shock discontinuity is typically several times the interparticle separation.

Classical fluid mechanics, as exemplified by modern aerospace codes, has followed more typically the Eulerian approach, where one uses a fixed (or adaptive) mesh, which is often, but not necessarily, Cartesian. Flux conserving codes have been developed which integrate the equations efficiently and accurately. In the recent past shock capturing methods have been combined with these codes which can stably represent the density and entropy jumps at shocks within two or three cells. Two examples of this are the code written by Ryu et al. (1993) based on the total variation diminishing scheme (TVD, hereafter) and the code written by Bryan et al. (1993) based on Piecewise Parabolic Method (PPM, hereafter). They have a second-order accuracy in time in contrast to "COJ" (Cen 1992), which is first order. Spatially, PPM aims at third order accuracy, TVD second order and COJ first order. For given computational resources the Eulerian codes can treat far more cells than the SPH codes can treat particles, but the 
cells are, in the simplest applications, uniformly spaced rather than concentrated into the more interesting high density regions as occurs naturally in the SPH codes.

Altogether dozens of papers have been written using these various methods with various claims made for the accuracy of the methods. The sceptical reader may well ask what credence should be given to such claims? What is the accuracy of these codes? If given the same initial conditions, do the different methods arrive at the same results? If not, which are to be trusted more? Or, more appropriately, which are to be trusted more in which type of region? Finally do they all converge ultimately (at fixed resolution), with application of sufficient resources, to the same final state, and if so at what rate? It is to answer these questions, as objectively as possible, that this paper is being written.

\section{COMPARISON}

\subsection{Test Calculations}

We have calculated the adiabatic evolution of a purely baryonic universe but with an initial CDM power spectrum, using the five cosmological hydro codes described in $\S 1$. The purpose of such a test calculation is to focus primarily on the hydrodynamic properties of these codes (and not, for example, on their gravity solvers). The random Gaussian initial conditions have been generated from the initial power spectrum using the process described in Weinberg \& Gunn (1990) with the normalization that the mass fluctuation in a sphere of radius $8 h^{-1} \mathrm{Mpc}$ is $\sigma_{8}$. The values of the usual cosmological parameters are $\Omega=\Omega_{b}=1, h=0.5$, and $\sigma_{8}=1$, and the size of the computational cube is $L=64 h^{-1} \mathrm{Mpc}$. The universe has been evolved from $z=20$ to $z=0$. For Eulerian codes (COJ, TVD, and PPM) simulations with $32^{3}, 64^{3}, 128^{3}$ and $256^{3}$ cells were performed. For SPH codes $32^{3}$ and $64^{3}$ particles are used. We generated the grid 
data from the SPH particle data by first smoothing over the interparticle scale and then integrating the smoothed data over the Eulerian grid cells.

The comparisons are made at the final epoch, $z=0$. The results at all these resolutions are rebinned onto a $16^{3}$ grid in order to make comparisons of the averaged quantities at a common coarse resolution. We have also made some comparisons using the higher resolution original data. Hereafter, we will adopt the notation for the simulations in such a way that TVD128, for example, means TVD $128^{3}$ simulation either at full resolution or as rebinned to a $16^{3}$ grid. The simulation with the COJ code was carried out by Cen, the TVD code by Kang, the PPM code by Bryan, the TSPH code by Hernquist and the PSPH code by Evrard. The required computational resources used for each code are given in Table 1. The hours of computer time listed in column 6 (in units of hours on a single processor Cray-YMP) are rough estimates. The listed error is from the Layzer-Irvine cosmological energy conservation equation and follows the prescription for $\epsilon=100|1-R|$ with the latter as defined by Eq. (3.10) of Ryu et al. (1993). Note that the $64^{3} \mathrm{SPH}$ codes require comparable or slightly more time $\left(\sim 10^{2}\right.$ hours) than the $256^{3}$ Eulerian codes ( 10 1.5 hours), but considerably less memory $\left(10^{-1}-10^{-1.5}\right.$ as much) to achieve a comparable $(c f ., \S 2.2)$ overall accuracy for results rebinned to low resolution.

\subsection{Comparison of Rebinned Data on a $16^{3}$ Grid}

First let us look at integral properties of the results. We have calculated the average temperature, $\langle T\rangle$, the average temperature weighted by the density, $\langle T\rangle_{\rho} \equiv\langle\rho T\rangle /\langle\rho\rangle$, the average temperature weighted by the density squared, $\langle T\rangle_{\rho^{2}} \equiv\left\langle\rho^{2} T\right\rangle /\left\langle\rho^{2}\right\rangle$, and the rms density fluctuation, $\sigma^{2} \equiv\left(\left\langle\rho^{2}\right\rangle /\langle\rho\rangle^{2}-1\right)$, on the data rebinned to a $16^{3}$ grid.

We also calculated the total X-ray luminosity, $L_{x} \equiv \Sigma \rho^{2} T^{1 / 2}$ (in arbitrary units) over the $16^{3}$ grid. Because of the coarse smoothing of the density field, this measure of $L_{x}$ underestimates by large factors the true luminosity. These quantities are summarized 
in Table 2. We plot $\langle T\rangle,\langle T\rangle_{\rho}, L_{x}$, and $\sigma$ versus the cube root of the number of the cells or particles in Fig. 1. Table 2 and Fig. 1 provide good measures of the rate of convergence of the simulations in terms of the shocked high density regions, but they are insensitive to the distribution of the unshocked low density IGM. According to Table 2, the PSPH32 and PSPH64 runs, for example, seem to be converged well with regard to density. But this does not necessarily mean, for example, that PSPH64 is more correct in all respects than the other methods, because the tabulated parameters are not much affected by the low density region where there are only small number of particles.

In general both SPH code runs have slightly higher temperatures than the gridbased Eulerian codes. At low resolution the difference is significant but we see in Table 2 that the high resolution codes converge well in the high temperature high density regions. Specifically the (density) ${ }^{2}$ average of the temperature is $(55.3,51.9,58.0,55.0)$ in the highest resolution runs of the (TVD,PPM,TSPH,PSPH) codes respectively, corresponding to a mean of 55.0 with variance 2.5 or only $4.5 \%$. With regard to this variable the Eulerian codes appear to converge from below (they underestimate $\langle T\rangle$ ) as does PSPH while for and TSPH the situation is less clear. The Jameson code (COJ) has poorer resolution than the TVD or PPM codes, since it is a first-order method, while the other two codes are based on higher order schemes. We note from Table 1 that it (COJ) is correspondingly more economical with regard to both memory and CPU requirements. The highest level of density fluctuation as rebinned to $16^{3}$ seems to be achieved in the TVD256 simulation $(\sigma=2.80)$, even though the PPM runs with $N<256$ have generally higher resolution than the comparable TVD runs (and we will see in 3b and $6 \mathrm{c}$ that in unbinned high resolution results TVD and PPM are almost identical). The average temperature seems to show a very good convergence for TVD256 run, while the density and the density weighted temperature indicate that somewhat more than $256^{3}$ cells would be required for the convergence of the density and pressure structure. 
A more detailed and definite set of comparison can be made on a cell-by-cell basis by comparing the density, temperature and pressure at each cell of the $16^{3}$ grids of two different simulations. The density ratios, for example, are calculated by the density of a "test" run divided by that of the "control" run at each cell. The ratios are grouped into bins according to the density of the "control" run. Then we calculated the median and first and third quartiles of the distribution of the logarithm of the ratios in each density bin of the "control" run. Fig. 2a shows the median of the logarithm of the density ratios for each code with TVD256 being picked arbitrarily as the "control" run. The displayed error bars are constructed from the differences between first or third quartiles and the median.

The density distributions of the three Eulerian codes are in a good agreement as seen in the left hand panels of (2a). But the density in the SPH codes in the low density regions tends to be higher than that of the TVD256 and to have larger scatter. This is true also for unbinned original data and is probably due to the small number of $\mathrm{SPH}$ particles in the low density regions. A sign that this may be attributed to error of the SPH code (in this coarse comparison) is that the higher resolution TSPH64 run is closer to the TVD256 run than is the TSPH32 run, indicating convergence towards the Eulerian result (in this comparison). The trend in the low density regions is approximately reversed if the "test" and "control" runs switch their places. The interpretation of the high density regions, however, is somewhat complicated, because the number density of cells with high density $(\rho / \bar{\rho}>1)$ decreases with the density and so the probability of spatial overlapping of high density cells between two codes becomes smaller. Suppose we take the ratios of density around two high density clusters which are similar in shape, but the locations of the clusters in the two simulations are slightly different. The ratio is more likely smaller than one for higher density bin, while it is more likely greater than one for lower density bin, because of the selection effect of the 
binning according to the density of the control run. This mismatching effect dominates in most of cases in Fig. 2a except perhaps $\rho(C O J 256) / \rho(T V D 256)$ versus $\rho(T V D 256)$.

Another, more impartial way to look at the high density regions is to plot a scatter diagram of $\log \left(\rho_{1} / \rho_{2}\right)$ versus $\log \left(\rho_{1} \rho_{2}\right)^{1 / 2}$ as in Fig. 2 b where $\rho_{2}=\rho(T V D 256)$. Except for $\rho(C O J 256) / \rho(T V D 256)$ and $\rho(T V D 128) / \rho(T V D 256)$ (which have lowest resolution), the points are randomly scattered about the mean, indicating that there is no significant difference in the high density regions among runs with different codes as rebinned to $16^{3}$ resolution.

Now let us turn to the temperature variable, which is most sensitive to details of the hydrodynamic treatment. The ratios of the temperature versus the temperature of TVD256 are plotted in Fig. 2c. For all cases, the temperature for high temperature regions seems to agree fairly well among the various simulations with the agreement beween the two high resolution codes (TVD, PPM: bottom left panel) quite good. As noted, the SPH values of temperature are slightly higher than the Eulerian values in the high density regions. The temperature of COJ256 for low temperature regions is higher than that of TVD256, because the COJ code has more artificial viscosity in low temperature regions with supersonic bulk motion. On the other hand, the temperature of the PPM run for low temperature regions is somewhat lower than that of TVD256.

The similar plots for the temperature ratios versus the gas density of TVD256 are shown in Fig. 2d. This shows that the temperature of high density regions is quite similar for all codes, but that of low density region displays significant differences. These results are consistent with Fig. 2c, since the high density cells also tend to have a high temperature.

Figs. 3a-b show the volume and mass weighted histograms of the density in $16^{3}$ cells for TVD256, PPM256, TSPH64, and PSPH64. The TVD256 and PPM256 runs have somewhat broader distributions than the SPH runs, because they have more lower density cells and more higher density cells. The agreement between the two Eulerian 
codes and between the two SPH codes is excellent. Fig. 3c-d shows the volume and mass weighted histograms of the temperature for the same cases as in Fig. 3a,b. Both SPH codes have somewhat more hot gas than TVD256 or PPM256. As expected, the volume occupied by the high temperature regions with $T \gtrsim 10^{6} \mathrm{~K}$ are similar in PPM and TVD codes.

Figs. $4 \mathrm{a}$ and $4 \mathrm{~b}$ show the density and temperature contour plots of a slice with $16 h^{-1} \mathrm{Mpc}$ thickness on $16^{3}$ grids in each the simulation. The density was normalized by the mean baryon density, while the temperature was normalized by $T_{b}=10^{6} \mathrm{~K}$ before contouring. The density contours show that the density distributions on a $16^{3}$ grid are, in general, quite similar for all codes. The temperature contours, on the contrary, show some differences. The SPH runs show more hot gas than the Eulerian code runs and it is spread out over larger regions perhaps due to less sharp shocks in low density regions. The temperature in COJ256 run is lower than than in TVD256 or PPM256, which is true even when compared with TVD128 or PPM128. This illustrates that, while the density structure seems to agree well, the temperature distributions shows much bigger differences among various hydro codes even after averaging the data onto a very coarse grid.

\subsection{Comparison of the Original Data}

Figs. 5a-c show the density and temperature contour plots of a slice with $0.25 h^{-1} \mathrm{Mpc}$ thickness (1 cell) on $256^{3}$ grids for three Eulerian codes, while Fig. 5 d shows the similar contour plots of a slice with $0.32 h^{-1} \mathrm{Mpc}$ for PSPH64 data mapped onto $200^{3}$ cells; and Figure (5e) shows a slice of $0.5 h^{-1} \mathrm{Mpc}$ smoothed onto a $256^{3}$ grid for the TSPH code result. The temperature contour plot for the COJ256 run indicates the artificial heating of the low density IGM. The agreement between the two high resolution Eulerian codes $[(\mathrm{PPM} 256, \mathrm{TVD} 256)=(5 \mathrm{~b}, 5 \mathrm{c})]$ is excellent. One sees in the temperature plots caustics emanating out of cluster like regions bounded by sharp shocks. From the thickness of 
the pancakes and the filaments (compared to the cell size), it is clear that the detailed structure has not converged even in $256^{3}$ run.

In Figs. 6a-e the contour plots of the cells (for Eulerian codes) or particles (for SPH codes) with given temperature and density are shown for three Eulerian and two SPH codes. Cells are weighted by mass in the Eulerian codes. The regions with the high temperature $\left(T \gtrsim 10^{6} \mathrm{~K}\right)$ are quite similar with highest density and temperature reached in the SPH codes. As has been seen in the histograms in Figs. 4, the warm and cold gas regions do not agree well among the four codes.

We calculated the fraction of the mass with given temperature and density for the TVD256 and PPM256 original data. The same fraction was calculated for the PSPH64 and TSPH64, using the original particle density and temperature, and counting particle numbers instead of mapping the particles onto a grid. They are plotted in Figs. 7b with the analogous Eulerian histograms shown in 7a. From these presentations, we can see that particle density in the TSPH32 and PSPH64 can become much larger than the grid density in the TVD256 in the high density postshock regions. On the other hand, the particle temperature distribution in the TSPH64 and PSPH64 is relatively close to that in the TVD256 and PPM256.

The different trends between the density and temperature can be understood simply, because the temperature is a conserved quantity (e.g. thermal energy per mass) within a particle, while the density is not (e.g. mass per volume). One of the obvious results is that the X-ray emissivity of the TSPH64 and PSPH64 particle data will be much greater than the TVD256, while the X-ray emissivity on $16^{3}$ resolution, $L_{x}$, in Table 2 of the three codes are quite close. They also show dramatically that the density is overestimated in the low density regions of the SPH calculation $(0.2<\rho / \bar{\rho}<5$ in Fig. 8). In the SPH results there are no particles with $\rho / \bar{\rho}<0.2$, while TVD256 has cells with density as low as 0.01 . One should note that, with the same number of particles, the TSPH64 run seems to achieve a slightly higher resolution than PSPH64. It is likely 
that the difference comes from the way with which the smoothing length varies in time and space in the two codes.

Finally we plotted the density and temperature distribution along an arbitrary line of sight for the TVD256 and PSPH64 in Figs. 8. The particles within a cylinder with a square face of $\left(1 h^{-1} M p c\right)^{2}$ along the line of sight are chosen for the PSPH64 and plotted as open circles. Among 16 rows of cells of TVD256 within the same square-faced cylinder, two at the corner and two near the center are shown. We can see the density of clusters in PSPH64 can become much higher than in TVD256, while the temperatures of clusters in the two runs are very similar. Once again, the X-ray appearance of the clusters in the two codes will be very different. This figure also illustrates well that only a small number of particles are in the low density region to represent the flow in the SPH code, so the density is overestimated in that region. Fig. 8b shows a blow-up of a single density peak around $R=33 h^{-1} \mathrm{Mpc}$ to examine in close-up the details of the high density cluster. There could be several explanations for the stronger clustering in PSPH64 than in TVD256 two of which are the following: 1) it could come from the difference between $\mathrm{P}^{3} \mathrm{M}$ code and Eulerian code for the gravitational field calculation since the SPH codes clearly have higher force resolution in dense regions; 2) it is possible that PSPH64 achieves hydrodynamically higher resolution than TVD256 due to its Lagrangian nature. Further investigation is necessary to discriminate among these possibilities.

\section{IMPLICATIONS FOR FUTURE WORKS}

It is reassuring to note in Fig. 1 that the various codes do in fact converge to the same results as $N \rightarrow \infty$. It appears that all codes converge from below with regard to density $\left(\left\langle\rho^{2}\right\rangle /\langle\rho\rangle\right.$ is always underestimated), but that with regard to the mean temperature the situation is not as clear. The Eulerian codes appear to converge from below as does PSPH while TSPH code may converge from above (or may not do so, 
but seem to in Table 1 due to our smoothing and rebinning procedures). With regard to the integral quantities, derived from rebinned data, it appears that $64^{3} \mathrm{SPH}$ results are of an accuracy intermediate between $128^{3}$ and $256^{3}$ in the shock capturing Eulerian codes. This indicates quite crudely (after examining Table 1) that by these measures comparable accuracy is reached with comparable computer time. The overall accuracy, as measured by convergence, is perhaps disappointing. Fig. 1 indicates roughly that some integrated quantities are still insecure to $20 \%$ in rebinned data (on a $16^{3}$ grid) for $N=64^{3} \mathrm{SPH}$ runs and the comparably costly Eulerian codes whereas other quantities have converged to $5 \%$.

Overall, the agreement in density, if one compares on a cell by cell basis, is far better than the agreement in temperature, especially in the high density regions. The SPH codes have poor information in the low density regions and poorer resolution of shocks but much better resolution in high density regions for comparable expenditure of computer resources. Agreement of temperature among the simulations in the high temperature regions is fairly good (given the caveats mentioned previously). In the low density $(\rho / \bar{\rho}<1)$, low temperature regions it appears that there is a quite significant overestimate of temperature by the COJ code.

One interesting fact is that in the binned data the distributions of temperature and density are broader in the Eulerian codes than in the SPH codes but in the unbinned data the converse is true. It is likely but not certain that the SPH codes give a better representation of the highest density regions due to their intrinsically Lagrangian nature, while the Eulerian codes are probably better in the regions $\rho / \bar{\rho}<1$ due to better sampling. The temperatures in regions like the X-ray clusters can be obtained with moderate accuracy with any of the highly developed codes, but there is still great uncertainty in computing the thermal energy of the lower temperature regions, This could be a problem in cosmological applications because, if the box scale is chosen to correspond to a wavelength bigger than that at the peak of the power spectrum, the 
"low" temperatures correspond to those in regions where galaxy formation is thought to occur.

These insights should lead us towards future code improvements, to better estimate of our accuracy and towards applications of the individual codes to problems for which they are best suited.

\section{Acknowledgments}

The work by HK, JPO, RC, and DR was supported in part by NASA through grant NAGW-2448 and by NSF through grants AST91-08103 and ASC-9318185. HK was supported in part by the Korea Research Foundation through the Brain Pool Program. LH was supported in part by NASA through grant NAGW-2422 and by NSF through Presidential Faculty Fellowship. AEE was supported in part by NASA through grant NAGW-2367. GLB and MLN was supported in part by NASA through NAGW-3152. GLB would like to thank Jim Stone for useful discussions. In the later stages of this work the Princeton, UCSC and NCSA work was supported by the NSF, HPCC grant ASC93-18185. 


\section{TABLE 1}

Required Computational Resources

\begin{tabular}{|ccrcccc|}
\hline run & Memory $^{\mathrm{a}}$ & $\mathrm{CPU}^{\mathrm{b}}$ (machine) & CPU $^{\mathrm{c}}$ & steps & totalCPU $^{\mathrm{d}} \epsilon(\%)$ \\
\hline TVD128 & 34 & 590 (Convex 220) & 59 & 130 & 2.1 & 4 \\
TVD256 & 250 & 4390 (Convex 3440) & 450 & 153 & 19.1 & 5 \\
\hline PPM128 & 32 & 180 (Convex 3880) & 90 & 200 & 7.2 & 9 \\
PPM256 & 190 & 169 (64-node CM5) & 559 & 255 & 57.6 & 4 \\
\hline COJ128 & 16 & 103 (Convex 220) & 14 & 490 & 1.9 & 2 \\
COJ256 & 128 & 1000 (Convex 3440) & 100 & 500 & 13.9 & 1 \\
\hline TSPH32 & 5 & 45 (Cray YMP) & 45 & 500 & 6.26 & 4 \\
TSPH64 & 40 & 360 (Cray YMP) & 360 & 500 & 50.0 & 3 \\
\hline PSPH32 & 0.94 & 240 (Stardent Titan 3020) & 12 & 1000 & 3.3 & 5 \\
PSPH64 & 7.5 & 73.8 (HP 735) & 144 & 1000 & 40 & 3 \\
\hline
\end{tabular}

${ }^{a}$ in units of Megawords

${ }^{b} \mathrm{CPU}$ time in units of seconds per time step

${ }^{c}$ equivalent $\mathrm{CPU}$ time on a single processor Cray-YMP in units of seconds per time step

${ }^{d}$ equivalent total CPU time on a single processor Cray-YMP in units of hour 
TABLE 2

Integrated Numbers

\begin{tabular}{|cccccc|}
\hline run & $\langle T\rangle^{a}$ & $\langle T\rangle_{\rho}^{a}$ & $\langle T\rangle_{\rho^{2}}^{a}$ & $\sigma$ & $L_{x}$ \\
\hline TVD32 & 0.720 & 3.71 & 13.1 & 1.52 & 0.419 \\
TVD64 & 1.53 & 9.79 & 30.2 & 2.23 & 1.20 \\
TVD128 & 1.83 & 13.1 & 42.5 & 2.58 & 1.86 \\
TVD256 & 1.85 & 16.2 & 55.3 & 2.80 & 2.45 \\
\hline PPM32 & 1.05 & 7.31 & 24.1 & 2.15 & 0.984 \\
PPM64 & 1.59 & 11.3 & 34.4 & 2.38 & 1.44 \\
PPM128 & 1.84 & 15.1 & 44.9 & 2.58 & 1.91 \\
PPM256 & 2.01 & 17.7 & 51.9 & 2.70 & 2.24 \\
\hline COJ32 & 0.519 & 2.26 & 6.56 & 1.52 & 0.302 \\
COJ64 & 1.19 & 6.21 & 18.2 & 1.93 & 0.733 \\
COJ128 & 1.44 & 8.86 & 26.7 & 2.25 & 1.16 \\
COJ256 & 1.57 & 11.9 & 38.2 & 2.49 & 1.65 \\
\hline TSPH32 & 2.92 & 24.4 & 68.4 & 2.70 & 2.61 \\
TSPH64 & 2.52 & 19.8 & 58.0 & 2.65 & 2.30 \\
\hline PSPH32 & 2.37 & 18.0 & 54.5 & 2.54 & 2.08 \\
PSPH64 & 2.46 & 19.2 & 55.0 & 2.67 & 2.27 \\
\hline
\end{tabular}

${ }^{a}$ in units of $10^{6} \mathrm{~K}$. 


\section{REFERENCES}

Barns, J., \& Hut, P. 1986, Nature, 324, 446.

Bertschinger, E., \& Gelb, J. 1991, Comput. in Phys., 5, 164.

Brainerd, T., \& Villumsen, J. 1992, ApJ, 394, 409.

Bryan, G. L., Norman, M. L., Stone, J. M., Cen, R., \& Ostriker, J. P. 1993, Computer Physics Communications, , in preparation.

Cen, R. 1992, ApJS, 78, 341.

Centrella, J. M., Gallagher, J. S., Melott, A. L., \& Bushouse, H. A. 1988, ApJ, 333, 24.

Davis, M., Efstathiou, G., Frenk, C. S., \& White, S. D. M. 1985a, ApJ, 292, 371.

Efstathiou, G., Davis, M., Frenk, C. S., \& White, S. D. M. 1985b, ApJS, 57, 241.

Efstathiou, G., \& Eastwood, J.W. 1981, MNRAS, 194, 503.

Evrard, A. E. 1988, MNRAS, 235, 911.

Hernquist, L. \& Katz, N. S. 1989, ApJS, 64, 715.

Ryu, D., Ostriker, J. P., Kang, H., \& Cen, R. 1993, ApJ, 414, 1.

Weinberg, D. H., \& Gunn, J. E. 1990, MNRAS, 247, 260. 


\section{FIGURE CAPTIONS}

Fig. 1.- Convergence of global averages. Average temperature (top left), average temperature weighted by the density (top right), the total X-ray luminosity (bottom left), and the rms density fluctuation $\left(\sigma^{2} \equiv\left\langle\rho^{2}\right\rangle /\langle\rho\rangle^{2}-1\right)$ of the rebinned data on a $16^{3}$ grid for the COJ (filled square), PPM (filled triangle), TVD (filled circle), PSPH (open circle), and TSPH (open triangle) codes as a function of the cube root of the number of cells or particles.

Fig. 2a. $-\log \left(\rho_{1} / \rho_{2}\right)$ versus $\log \left(\rho_{2}\right)$ where $\rho_{2}=\rho($ TVD256). The simulation designations for $\rho_{1}$ are expressed in the labels on the abscissa. The solid line is the median of the logarithm of the density ratio distribution at each bin, while the error bars are constructed from the differences between first and third quartiles and the median. Bottom left panel shows that high resolution Eulerian codes agree well with one another, and comparison of two lower right hand panels shows that the two SPH codes agree well with each other.

Fig. 2b. - $\log \left(\rho_{1} / \rho_{2}\right)$ versus $\log \left(\rho_{1} \rho_{2}\right)$ for high density cells on a $16^{3}$ grid. For all cases $\rho_{2}=\rho(T V D 256)$ as in the Fig. 2a. The simulation designations for $\rho_{1}$ are expressed in the labels on the abscissa. No trends are seen, indicating that in the rebinned data all codes have similar resolution except COJ256 and TVD128 which reach systematically lower densities in high density regions.

Fig. 2c.- Same as Fig. 2a except the temperature ratios are plotted for the temperature of the TVD256 run. Note agreement in the high temperature regions and variance in the low temperature regions.

Fig. 2d.- Same as Fig. 2c except the temperature ratios are plotted for the density of the TVD256 run. Note agreement in the high density regions and variance in low density regions. 
Fig. 3a.- Mass weighted density histogram for the TVD256 (top panel, solid line), PPM256 (top panel, dashed line), TSPH32 (bottom panel, solid line) and PSPH64 (bottom panel, dashed line) runs. The simulation results at $z=0$ rebinned onto a $16^{3}$ grid are shown. Eulerian codes show a slightly broader distribution.

Fig. 3b.- Volume weighted density histogram for the same simulations as in Fig. 3a.

Fig. 3c. - Mass weighted temperature histogram for the same simulations as in Fig. 3a. SPH codes reach somewhat higher temperatures.

Fig. 3d.- Volume weighted temperature histogram for the same simulations as in Fig. 3a. Peaks in very low temperature regions are spurious, numerical artifacts.

Fig. 4a.- Density contour plots of a slice with $16 h^{-1} \mathrm{Mpc}$ thickness on a $16^{3}$ grid for each simulation. General agreement is seen.

Fig. 4b.- Same as Fig. 4a except temperature contour plots are shown. SPH codes show broader peaks possibly due to poorer resolution of shocks.

Fig. 5a.- Gas density (top) and temperature (bottom) contour plots of a slice with $0.25 h^{-1} \mathrm{Mpc}$ thickness on a $256^{3}$ grid for the COJ256 run. Note in (5a-5d) the familiar pattern of caustics connecting vertices of high temperature gas.

Fig. 5b.- Same as Fig. 6a except for the PPM256 run.

Fig. 5c. - Same as Fig. 6a except for the TVD256 run.

Fig. 5d.- Gas density (top) and temperature (bottom) contour plots of a slice with $0.32 h^{-1} \mathrm{Mpc}$ thickness on a $200^{3}$ grid for the PSPH64 run. The location of the slice is the same as the ones in Fig. 5c.

Fig. 5e.- Gas density (top) and temperature (bottom) contour plots of a slice with $0.5 h^{-1} \mathrm{Mpc}$ thickness on a $256^{3}$ grid for the TSPH64 run. The location of the slice is the same as the ones in Fig. 5c

Fig. 6.- Contour plot of the volume fraction with given temperature and density. The simulation results at $z=0$ on a $128^{3}$ grid are shown. The plots are for (a) COJ256 
(b) PPM256, (c) TVD128, (d) PSHP64, and (e) TSPH64. SPH codes reach to higher temperatures and densities. See text for binning/smoothing parameters. Fig. 7a.- Histograms of the mass fraction with given temperature and density for TVD256 (solid line) and PPM256 (dashed line) original data.

Fig. 7b. - Histograms of the mass fraction with given temperature and density for TSPH64 (dashed line) and PSPH64 (solid line) original data.

Fig. 8a.- Density and temperature distribution along an arbitrary line of sight for the TVD256 original data (left two panels) and the PSPH64 original particle data (right two panels). Four different rows of cells in the TVD256 run are shown within a cylinder with a square face of $\left(1 h^{-1} \mathrm{Mpc}\right)^{2}$, while the particles of the PSPH64 run located in the same cylinder are plotted.

Fig. 8b.- Same as Fig. 9a except that blow up of a single peak is shown. 